\title{
Performance Evaluation of Modified SVD based Image Fusion
}

\author{
Asha P Kurian, Bijitha S R, Lekshmi Mohan, Megha M Kartha, K P Soman \\ Centre For Excellence in Computational Engineering and Networking \\ Amrita Vishwa Vidyapeetham, Amrita School of Engineering, Coimbatore-641112
}

\begin{abstract}
Image fusion produces a single composite image from a set of input images, which is more suitable for visual perception and computer processing. This paper proposes Singular Value Decomposition (SVD) based fusion, which yields better results. The variation of data can be captured by SVD and is used to perform the fusion by varying singular values. This approach is implemented and compared with image fusion based on PCA (Principal component analysis) and performance is evaluated using various image quality measures such as PSNR, Normalized cross correlation, Structured content, MSE, Normalized absolute error. Simulation results of proposed approach shows significant performance improvement when compared with PCA based fusion.
\end{abstract}

\section{Keywords}

Image fusion, PCA, SVD

\section{INTRODUCTION}

Image fusion is the process of combining the information from two or more images of same scene in to single image. This helps to achieve high spatial and spectral resolution in a single image. Image fusion can be performed at different levels such as signal level, pixel level, feature level and decision level [1]. The proposed approach is based on pixel level fusion. It generates a fused image in which information associated with each pixel is determined from a set of source images.

Image Fusion begins with the concept of simply averaging the intensities of the corresponding pixels of the input images and produces a fused image. A lot of advancements have happened in the field of image fusion by applying some techniques. The widely used image fusion techniques includes Intensity-Hue-Saturation (IHS), high pass filtering, principal component analysis (PCA), different arithmetic combinations, multiresolution analysis based methods (pyramid algorithm and wavelet transform), Artificial Neural Networks(ANN) etc [1]. Pyramid decomposition methods construct a fused pyramid from the pyramid representation of the original image but it had some disadvantages like blocking effects and lack of flexibility [2]. The limitations of HIS transform are that it distorts colours and it is limited to three colour bands (RGB) [3]. The principal component thermographic technique used to process the thermal image acquired from composite structure proposed in [4]. It is applied on flash thermographic inspection data of the composite structure. Image fusion algorithm using Finite Ridgelet Transform (FRIT) is used to decompose the row images to low and high frequency components [5,6]. A fusion method based on SVD was applied to multispectral face recognition [7]. The face images are decomposed in to three layers according to the energy distribution. SVD is applied in order to enhance the super resolution images by converting the reference image in to
SVD domain and the images' singular values are fused before performing interpolation [8].

In the proposed paper, SVD based image fusion was compared with PCA. SVD captures the variation of data and it is used to perform the fusion by various singular values. The approach is implemented and compared with image fusion based on PCA and performance is evaluated using various quality measures such as Peak Signal to Noise Ratio(PSNR), Normalized cross correlation(NC), Structured content(SC), Mean Squared Error(MSE), Normalized absolute error(NAE).

The rest of the paper is organized as follows. Section 2 introduces image fusion methods. The performance discriminators to determine the quality of image is explained in Section 3. The proposed method is introduced in Section 4. Simulation results are presented and discussed in Section 5 and concluded in Section 6.

\section{IMAGE FUSION METHODS}

Image fusion is a framework where a composite image can be produced which contains enhanced information using some methods. Here mainly PCA and SVD based fusion methods are taken in to consideration.

\subsection{PCA}

Principal Component Analysis (PCA) is a linear transformation technique for dimensionality reduction. It projects the data from original space to its eigen space to increase the variance by keeping the lower order principal components and ignoring higher order ones. Such lower order components often contain the most important aspects of data. It is widely used in data compression and pattern matching by expressing the data in a way to highlight the similarities and differences without much loss of information [9].

Derivation:

Consider a P-dimensional subspace containing n-tuple data where $\mathrm{P}<<\mathrm{n}$. Assume that the subspace is linear such that a set of linearly independent orthogonal basis vectors span the subspace $\left\{\beta_{1}, \beta_{2}, \beta_{3} \ldots \beta_{p}\right\}$ where each $\beta_{p} \in R^{n}$

So each data point $x$ can be approximated by a linear combination of these basis vectors.

$$
\begin{aligned}
& x \approx \sum_{p=1}^{p} r_{p} \beta_{p}=B r \quad \text { where } \mathrm{B} \text { is an nxp dimension matrix } \\
& \boldsymbol{B}=\left[\boldsymbol{\beta}_{1}, \boldsymbol{\beta}_{2}, \boldsymbol{\beta}_{3} \ldots \boldsymbol{\beta}_{p}\right] \text { and } \mathrm{r} \text { is a Px1 dimensional }
\end{aligned}
$$

vector.

Consider the limiting case where $\mathrm{P}=1$, the data $\mathrm{X}$ is modelled as residing around a one-dimensional linear subspace $\beta_{1}$ and assume that the data $\mathrm{X}$ has zero mean. The squared 
reconstruction error incurred when making the approximation $x \approx r_{1 i} \beta_{i}$ defined as

$$
\begin{aligned}
\varepsilon & =\frac{1}{m} \sum_{i=1}^{m}\left\|x_{i}-r_{1 i} \beta_{1}\right\|^{2}=\frac{1}{m} \sum_{i=1}^{m}\left(x_{i}^{T} x_{i}-2 r_{1 i} \beta_{1}^{T} x_{i}+r_{1 i}^{2} \beta_{1}^{T} \beta_{1}\right) \\
& =\frac{1}{m} \sum_{i=1}^{m}\left(x_{i}^{T} x_{i}-2 r_{1 i} \beta_{1}^{T} x_{i}+r_{1 i}{ }^{2}\right)
\end{aligned}
$$

Since $\beta_{1}^{T} \beta_{1}=1$

Now given $\beta_{1}$, we have to find what value of $r_{1 i}$ s will minimise the error .Taking derivatives with respect to each $u_{1 i}$ and setting to zero gives

$-2 \beta_{1}^{T} x_{i}+2 r_{1 i}=0 \Rightarrow r_{1 i}=\beta_{1}^{T} x_{i}$.

To get the vector $\beta_{1}$, substitute $r_{1 i}=\beta_{1}^{T} x_{i}$ in the equation for error.

$$
\varepsilon=\frac{1}{m} \sum_{i=1}^{m}\left\|x_{i}-r_{1 i} \beta_{1}\right\|^{2}=\frac{1}{m} \sum_{i=1}^{m}\left(x_{i}^{T} x_{i}-2 r_{1 i} \beta_{1}^{T} x_{i}+r_{1 i}^{2}\right)=\frac{1}{m} \sum_{i=1}^{m}\left(x_{i}^{T} x_{i}\right.
$$$$
=\frac{1}{m} \sum_{i=1}^{m}\left(x_{i}^{T} x_{i}-\left(\beta_{1}^{T} x_{i}\right)\left(\beta_{1}^{T} x_{i}\right)^{T}=\frac{1}{m} \sum_{i=1}^{m}\left(x_{i}^{T} x_{i}-\beta_{1}^{T} x_{i} x_{i}^{T} \beta_{1}\right)\right.
$$

Here $\frac{1}{m} \sum_{i=1}^{m} x_{i}^{T} x_{i}$ is a constant and it is equal to total variance. $\frac{1}{m} \sum_{i=1}^{m} r_{1 i}^{2}=\frac{1}{m} \sum_{i=1}^{m} \beta_{1}^{T} x_{i} x_{i}^{T} \beta_{1} \quad$ represents a variation of the projected values of $\beta_{1}$. So in order to minimise the reconstruction error, it is important to choose a $\beta_{1}$ value along which variance of the projected values is maximum.

$\max _{\beta_{1}} \frac{1}{m} \sum_{i=1}^{m} \beta_{1}^{T} x_{i} x_{i}^{T} \beta_{1} \quad$ subjected to $\beta_{1}^{T} \beta_{1}=1$

But $\frac{1}{m} \sum_{i=1}^{m} \beta_{1}^{T} x_{i} x_{i}^{T} \beta_{1}=\frac{1}{m} \sum_{i=1}^{m} \beta_{1}^{T} X X^{T} \beta_{1}=\beta_{1}^{T} \widehat{C} \beta_{1}$

where $\widehat{C}$ is the variance-covariance matrix of the variables $X_{1}, X_{2}, \ldots X_{n}$

In PCA based image fusion, arrange the source images in to two column vectors and compute empirical mean along each column. Then subtract the empirical mean from each column and find covariance matrix of resulting matrix. Compute eigen vectors and eigen values and sort them by decreasing eigen value [10]. Consider the first column of eigen vector which corresponds to larger eigen value to compute normalized component.

\subsection{SVD}

SVD is a technique which factorises matrix A into three matrices $u, v$ and $\sum$ such that $A=u \sum v^{T}$ According to

Pythagoras theorem the sum of the squares of the lengths of all data points is equal to the sum of squares of all the elements of the matrix A [11].

Consider two unit norm vectors $e_{1}=\left[\begin{array}{l}1 \\ 0\end{array}\right]$ and $e_{2}=\left[\begin{array}{l}0 \\ 1\end{array}\right]$

$A e_{1}$ and $A e_{2}$ represents $x_{1}$ and $x_{2}$ coordinates of all data points.

Let $\mathrm{S}$ represent the total variation of the data.

$$
S=\left(A e_{1}\right)^{T}\left(A e_{1}\right)+\left(A e_{2}\right)^{T}\left(A e_{2}\right)
$$

$S=e_{1}^{T} A^{T} A e_{1}+e_{2}^{T} A^{T} A e_{2}$

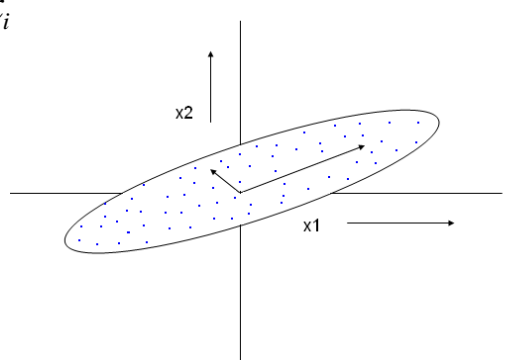

Fig 1-Geometrical approach of SVD [11]

The principal axis of the ellipse represents the direction along which data is distributed. The remaining variation is along an axis perpendicular to yhe principal axis. A 2-d vector can be decomposed into two components in any two orthogonal direction. Consider two orthogonal unit vectors $v_{1}$ and $v_{2}$ and project data onto those vectors. $S=v_{1}^{T} A^{T} A v_{1}+v_{2}^{T} A^{T} A v_{2}$

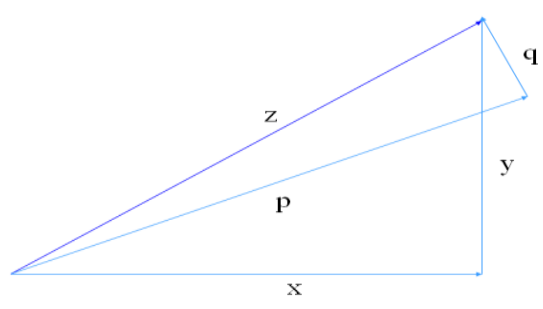

Fig 2-Pythagoras theorem [11]

$z^{2}=x^{2}+y^{2}$ and $z^{2}=p^{2}+q^{2}$ 
Consider two unit norm orthogonal vectors $v_{1}$ and $v_{2}$ such that maximum variation along $v_{1}$ axis which is at 45 degree to the $\mathrm{x}$ axis.

$v_{1}=\left[\begin{array}{l}1 / \sqrt{2} \\ 1 / \sqrt{2}\end{array}\right]$

The variation along this direction is

$\left(A v_{1}\right)^{T}\left(A v_{1}\right)=v_{1}^{T} A^{T} A v_{1}$

This is obtained by solving an optimization problem.

$\max v_{1}^{T} A^{T} A v_{1}$

subject to $v^{T} v=1$

Taking Lagrangian and applying first order optimization condition.

$$
\begin{aligned}
& L(v, \lambda)=v^{T} A^{T} A v-\lambda\left(v^{T} v-1\right) \\
& \frac{\partial L}{\partial v}=2 A^{T} A v-2 \lambda v=0 \Rightarrow A^{T} A v=\lambda v
\end{aligned}
$$

The direction is given by eigen vector of $A^{T} A$. Choose the eigen vector corresponding to largest eigen value $\lambda$ represent variation along direction $v$.

$$
v^{T} A^{T} A v=v^{T} \lambda v=\lambda v^{T} v=\lambda
$$

Let $A v_{1}=a_{1} u_{1} . A v$ represent the component of each data point. Similarly $A v_{2}=a_{2} u_{2}$.

$$
A\left[\begin{array}{ll}
v_{1} & v_{2}
\end{array}\right]=\left[\begin{array}{ll}
u_{1} & u_{2}
\end{array}\right]\left[\begin{array}{cc}
a_{1} & 0 \\
0 & a_{2}
\end{array}\right]
$$

$A V=U \sum$

Since the columns of $V$ are orthonormal, $V^{T} V=I$

$$
A=U \sum V^{T}=\left[\begin{array}{ll}
u_{1} & u_{2}
\end{array}\right]\left[\begin{array}{ll}
a_{1} & 0 \\
0 & a_{2}
\end{array}\right]\left[\begin{array}{l}
v_{1}^{T} \\
v_{2}^{T}
\end{array}\right]
$$

The dimensions of each matrix can be

$$
A_{m \times 2}=U_{m \times 2} \sum_{2 \times 2} V_{2 \times 2}^{T}
$$

Applications of SVD includes system identification [12], order determination [13], image coding [14] etc.

\section{PERFORMANCE EVALUATION}

The quality of the proposed image fusion method is evaluated based on certain parameters such as Peak Signal to Noise Ratio(PSNR), Normalized cross correlation, Structured content, Mean Squared Error(MSE), Normalized absolute error(NAE) [15]

Mean Squared Error (MSE): It is the average of the squares of errors. Error is the amount by which the value implied differs from the original value. The image quality decreases as MSE increases.

$$
M S E=\frac{1}{m n} \sum_{i=0}^{m-1} \sum_{j=0}^{n-1}[I(i, j)-K(i, j)]^{2}
$$

Peak Signal to Noise Ratio (PSNR): It is the ratio between the maximum possible power of a signal and the power of noise that affects the fidelity of the output. The image quality increases as PSNR increases.

$P S N R=20 \log _{10}\left(\frac{M A X_{i}}{\sqrt{M S E}}\right)$ where $M A X_{i}$ is the maximum possible pixel value of the image.

Normalized cross correlation (NC): Cross correlation is the measure of similarity of two images. In image processing applications the brightness of the image can vary due to exposure conditions, so images can be first normalized. The image quality increases as $\mathrm{NC}$ increases.

$$
N C=\frac{1}{n} \sum_{x, y} \frac{(f(x, y)-\bar{f})(t(x, y)-\bar{t})}{\sigma_{f} \sigma_{t}}
$$

where $\mathrm{n}$ is the number of pixels in $t(x, y)$ and $f(x, y)$, $\bar{f}$ is the average of $\mathrm{f}, \sigma_{f}$ and $\sigma_{t}$ are standard deviations

Structured Content (SC): It is the ratio between the content of the both the expected and the obtained data. Practically, it is the ratio between the net sum of the square of the expected data and the net sum of square of the obtained data.

$$
S C=\frac{\sum_{i=1}^{m} \sum_{j=1}^{n}\left(A_{i j}\right)^{2}}{\sum_{i=1}^{m} \sum_{j=1}^{n}\left(B_{i j}\right)^{2}}
$$

Where $m$ is the height of the Image implying the number or pixel rows, $n$ is the width of the image, implying the number of pixel columns. $A(i, j)$ being the pixel density values of the perfect image. $B(i, j)$ being the pixel density values of the fused image. The quality of the image increases while SC decreases.

Normalized absolute error (NAE): It is a metric where the error value is normalised with respect to the expected data. That is, the net sum ratio between the error values and the perfect values is calculated. The net sum of the error value which is the difference between the expected values and the actual obtained values is divided by the net sum of the expected values. 


$$
N A E=\frac{\sum_{i=1}^{m} \sum_{j=1}^{n}\left(\left|A_{i j}-B_{i j}\right|\right)}{\sum_{i=1}^{m} \sum_{j=1}^{n}\left(A_{i j}\right)}
$$

Where $m$ is the height of the Image implying the number or pixel rows, $n$ is the width of the image, implying the number of pixel columns. $A(i, j)$ being the pixel density values of the perfect image. $B(i, j)$ being the pixel density values of the fused image.

\section{PROPOSED METHOD}

In the proposed method, image fusion is achieved by applying singular value decomposition to two blurred input images. By combining the relevant information from these two images, a more informative and complete image can be produced. This is achieved by applying Singular value decomposition to the input images that would make the good information in each of the image prominent. In this method, image is decomposed into 3 components, $\mathrm{U}, \sum$ and $v^{T}$ which contains full information. In this, $U \& \mathrm{~V}$ are orthonormal matrices and $\Sigma$ represents a diagonal matrix which gives the variations of data points. Columns of $\mathrm{V}$ are eigen vectors of matrix $A^{T} A$. Columns of $\mathrm{U}$ are projections of $\mathrm{A}$ (or data points which are rows of A) on to columns of $\mathrm{V}$ vectors. $\Sigma$ is a diagonal matrix and diagonal elements are square root of variation of data points along the columns of V. By this approach fused output can be obtained by taking only certain components. Here, assigning different singular values means reduced number of components from each matrix $\mathrm{U}, \sum, \mathrm{V}$ by preserving the actual image size. After that image fusion is performed using this reduced number of components and thus obtained the fused output

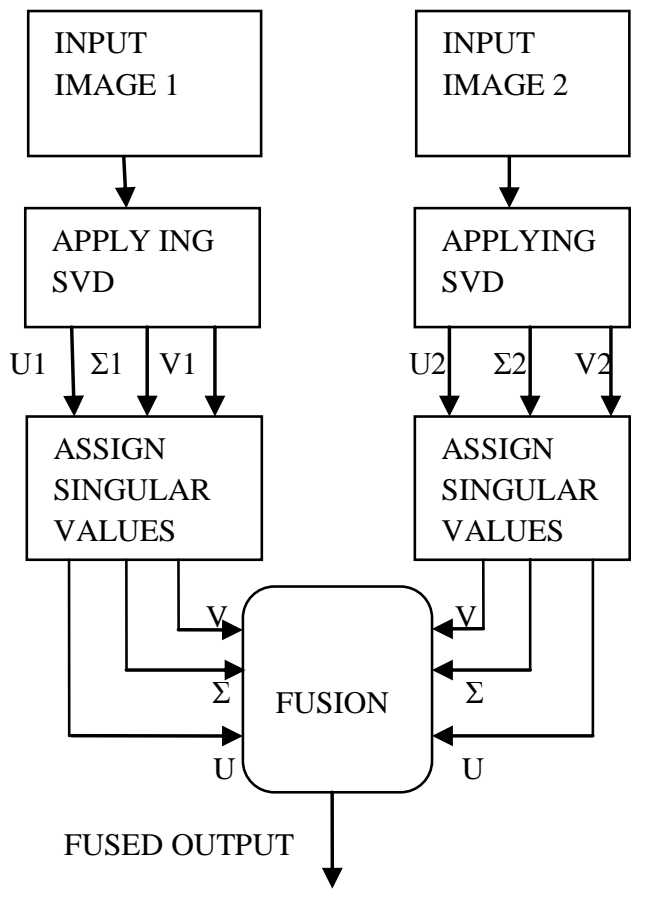

Fig-3 Block diagram of SVD based fusion

\section{RESULTS AND DISCUSSION}

In this section, the performance of proposed method of SVD based image fusion is tested using one standard data set which contains two blurred images. These results are compared with the results of fusion carried out by the method of PCA (Principle Component Analysis) on the same set of input images.

The SVD based approach is tested using different values of singular values, $\mathrm{k}$ as 10 to 150 . Table 2 shows the different image quality parameters for different values of $k$, i.e. for different singular values. On the other hand Table 1 shows comparison of image quality parameters of the proposed approach and PCA based image fusion with the same input image set.

Here Fig 4,5and6 shows the datasets which contains input images for fusion \& the reference image respectively.Fig-5.1.d to fig 10 illustrates the output images for different singular values from 10 to 150 . Fig 11 gives the image resulted from PCA based image fusion. Fig 12 to fig 16 shows plots of different image quality measures vs. singular values ranges from 10 to 150 . From input images it is understood that the different parts of input images are blurred, which reduces the clarity of the visual perception. The perfect image which is shown here, act as the reference image for deciding the image quality measures of the output image. Output images part displays the different output images for various singular values using the proposed SVD based fusion method. Based on these output images Table-1 is created, which gives the various image quality measures. From fig 7 to fig 10, it is understood that output image quality is changed according to the change in singular values. As the singular value is getting changed from 10 to 150 , the image quality has increased drastically. The detailed analysis can be done by using the table-2. In this, values of various image quality parameters as PSNR(peak signal to noise ratio),MSE(mean square error), $\mathrm{NCC}$ (normalised cross correlation), $\mathrm{SC}$ (structural content),\&NAE(normalised absolute error) are measured with respect to the reference image is given.

The value of PSNR is always high for good quality images. In the table it is seen that the PSNR value is 23.61 when singular value $(\mathrm{k})$ is 10 .It has reached 31.20 for $\mathrm{k}=150$.from this it can be concluded that the image quality is very high for higher values of $\mathrm{k}$. Similarly M.S.E which indicates the mean square error of two images should be very low for good visual images. Here M.S.E has changed from 282.5913 (for $\mathrm{k}=10$ ) to 49.28(for $\mathrm{k}=150$ ). This indicates that the error is very low for higher singular value and image quality of output image is high. When looking in to the case of NC, it is known that its value is quite high for good output images with respect to reference image. Here also it can be observed that, when the singular values has increased from $\mathrm{k}=10$ to 150 the value of $\mathrm{NC}$ also gets increased from 0.94 to 0.98 . This points towards the good image quality of the fused output. Similarly while considering the case of SC, it should be low for good quality images. In that way, here it is well understood that it is decreased from 1.05 (for $\mathrm{k}=10$ ) to 1.02 (fork=150).It indicates the good quality output image for $\mathrm{k}=150$.ie, for high singular value.

At last when we consider NAE, it is seen that it decreases from 0.1845 (for $\mathrm{k}=10$ ) to 0.0498 (for $\mathrm{k}=150$ ). Generally NAE should be low for high image quality. So it can conclude that the output fused image is of higher quality for higher singular value.

Thus from the above facts it can be concluded that the fusion of two input images by using SVD approach gives good 
quality outputs as the singular value increases. It is depicted in the plots given in Fig 12 to fig 16-, which indicates the variation of different parameters with singular value.

In fig 11 the output obtained by fusion via, PCA (principle component analysis) is given. Table 1 shows the values of image quality measures for the outputs obtained by PCA and SVD. In this it is seen that the value of PSNR is 31.20 where as for PCA it is only 29.0, where SVD gives good result as explained earlier. Then while discussing about MSE, it's value is 49.28 whereas for PCA it is 81.83 , which gives high error in the output. Similarly other parameters also indicate that SVD based fusion gives better results than PCA based fusion.

\subsection{Simulation results}

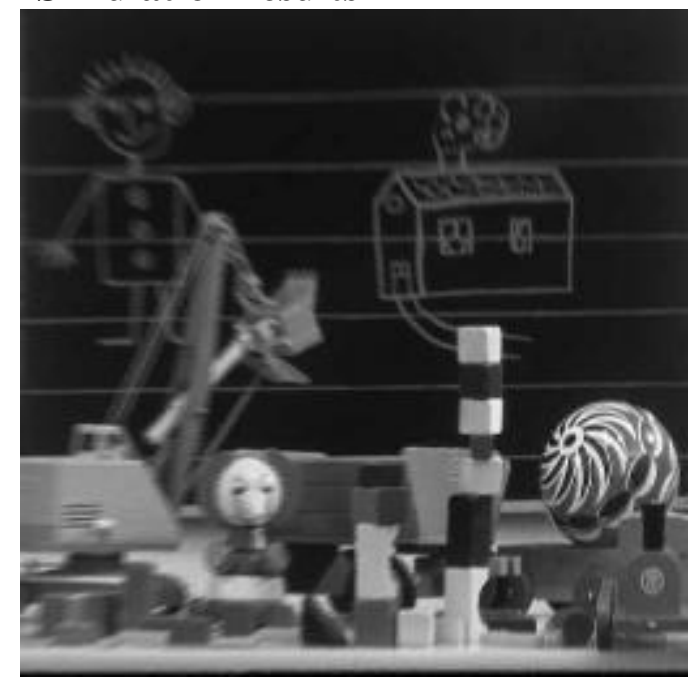

Fig 4 - Input image1

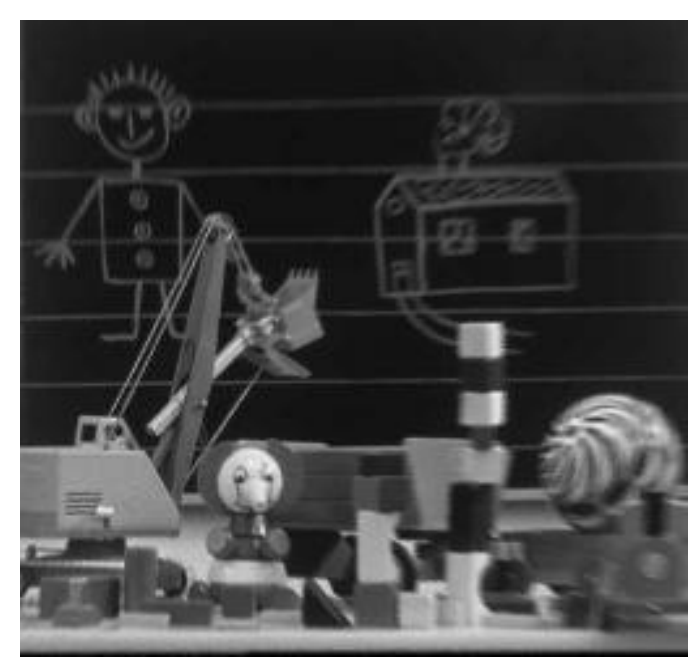

Fig 5- Input image2

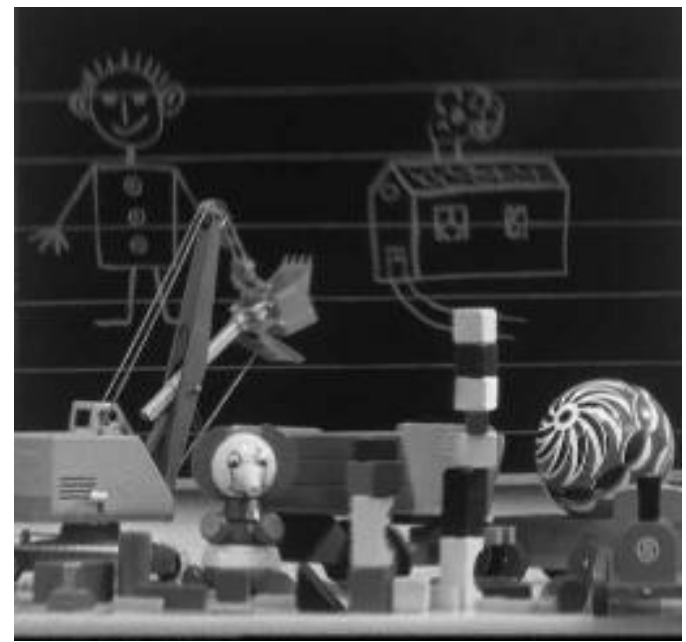

Fig 6-Reference image

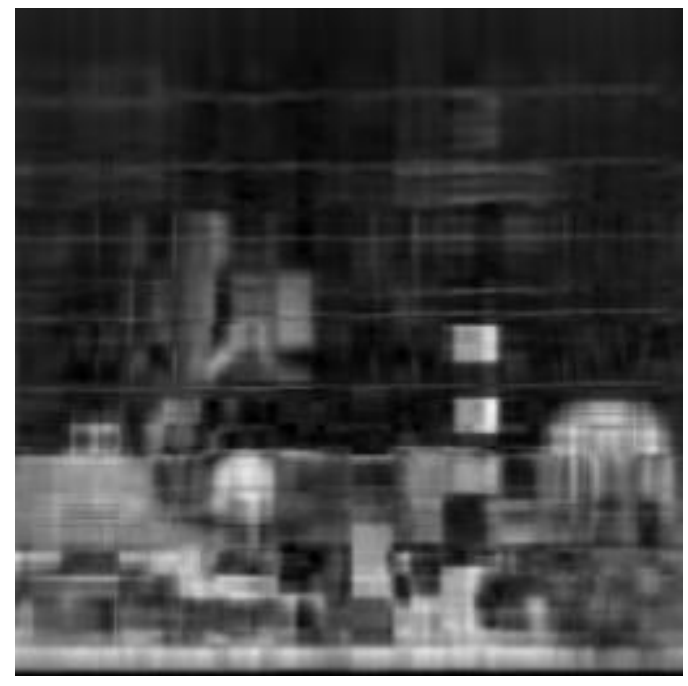

Fig 7 - Output image $(k=10)$

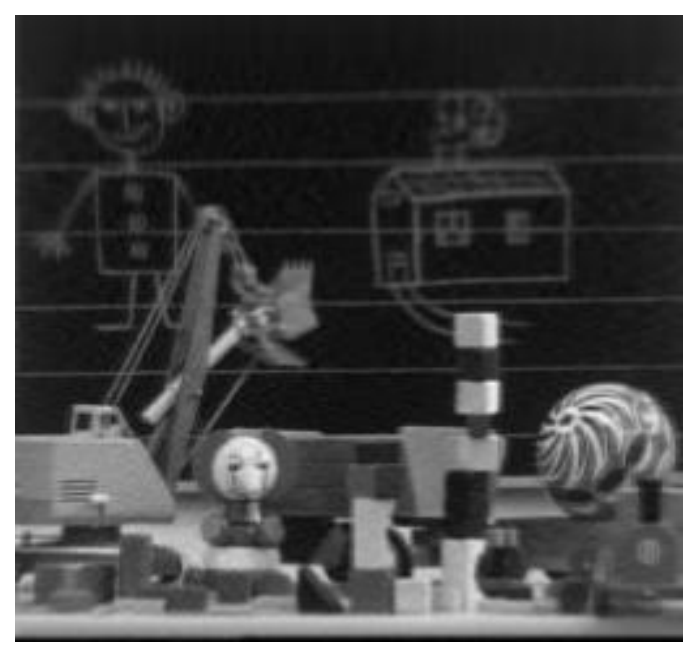

Fig 8- Output image $(k=50)$ 


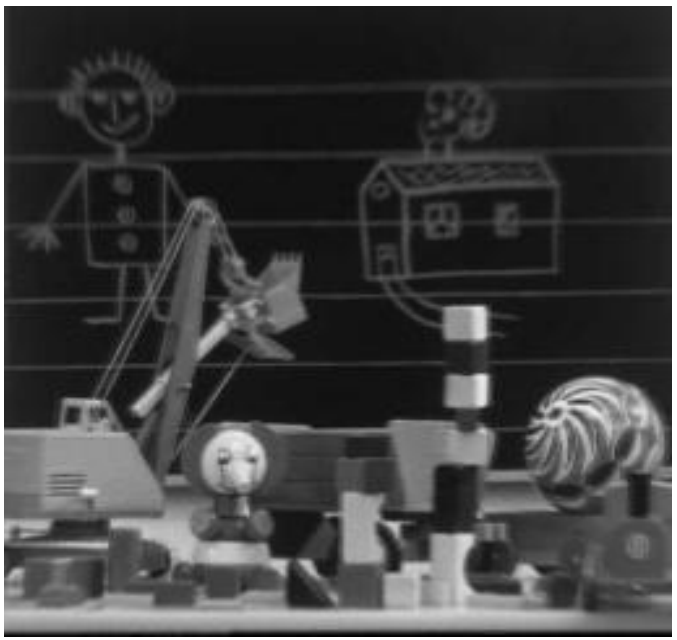

Fig 9- Output image $(k=100)$

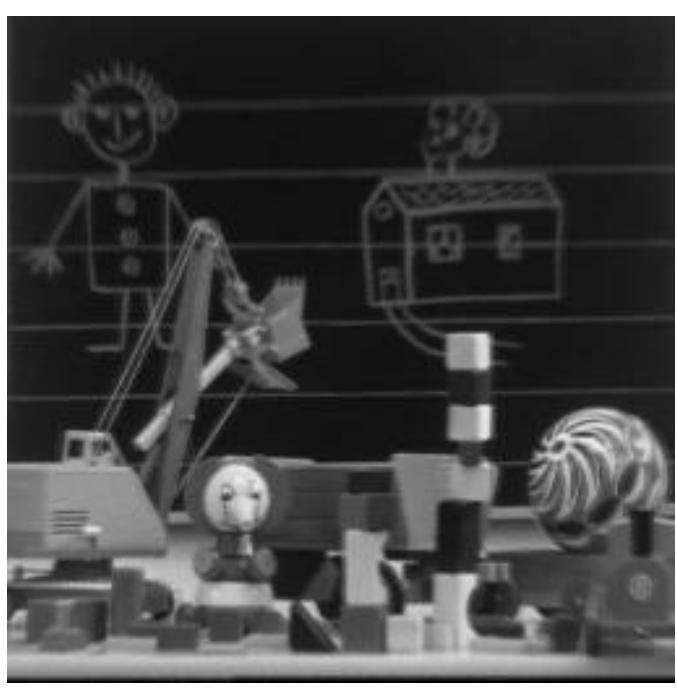

Fig 10- Output image $(k=150)$

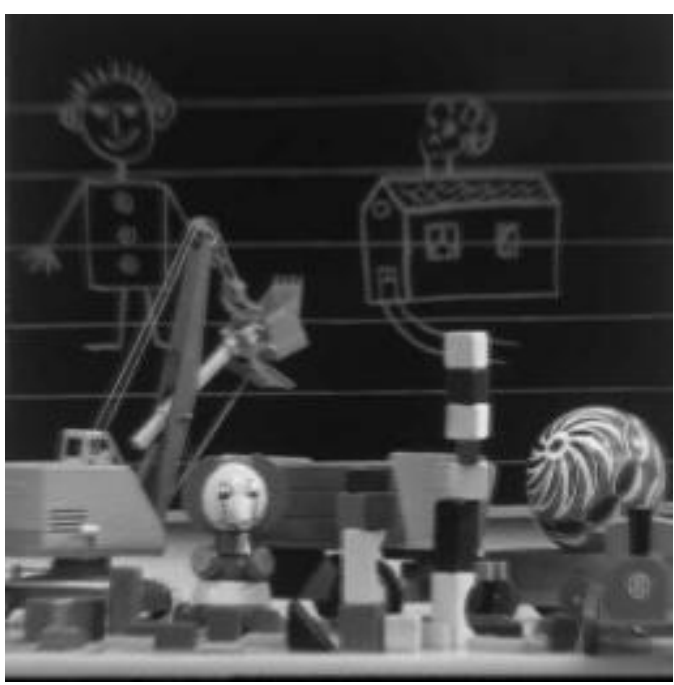

Fig 11- Output image for PCA based fusion

\subsection{Comparative study of outputs}

The performance discriminators of output images of SVD based fusion for different singular values are computed and plotted. Also the performance of SVD and PCA based fusion are compared and tabulated. The conclusion is made on the basis of these plots and tables

Table 1, Comparison of SVD and PCA Based Fusion

\begin{tabular}{|c|c|c|}
\hline Parameters & SVD with k=150 & PCA \\
\hline PSNR & 31.2037 & 29.00 \\
\hline MSE & 49.2847 & 81.83 \\
\hline NC & 0.9861 & 0.9780 \\
\hline SC & 1.0202 & 1.0315 \\
\hline NAE & 0.0498 & 0.0715 \\
\hline
\end{tabular}

Table 2, performance of SVD for different values of $k$

\begin{tabular}{|c|c|c|c|c|c|}
\hline K & PSNR & MSE & NC & SC & NAE \\
\hline 10 & 23.6192 & 282.5913 & 0.9498 & 1.0569 & 0.1845 \\
\hline 20 & 26.3863 & 149.4354 & 0.9711 & 1.0343 & 0.1277 \\
\hline 30 & 28.065 & 101.5234 & 0.9781 & 1.0277 & 0.1003 \\
\hline 40 & 29.1509 & 79.0669 & 0.9815 & 1.0245 & 0.0837 \\
\hline 50 & 29.9249 & 66.1588 & 0.9834 & 1.0228 & 0.0729 \\
\hline 60 & 30.4342 & 58.8377 & 0.9845 & 1.0217 & 0.0655 \\
\hline 70 & 30.7425 & 54.8065 & 0.9851 & 1.0211 & 0.0605 \\
\hline 80 & 30.9284 & 52.5091 & 0.9855 & 1.0208 & 0.0574 \\
\hline 90 & 31.0480 & 51.0835 & 0.9857 & 1.0205 & 0.0550 \\
\hline 100 & 31.1127 & 50.3286 & 0.9859 & 1.0204 & 0.0534 \\
\hline 110 & 31.1539 & 49.8534 & 0.9859 & 1.0203 & 0.0523 \\
\hline 120 & 31.1763 & 49.5969 & 0.9860 & 1.0202 & 0.0514 \\
\hline 130 & 31.1865 & 49.4795 & 0.9860 & 1.0202 & 0.0508 \\
\hline 140 & 31.1968 & 49.3625 & 0.9860 & 1.0202 & 0.0503 \\
\hline 150 & 31.2037 & 49.2847 & 0.9861 & 1.0202 & 0.0498 \\
\hline
\end{tabular}




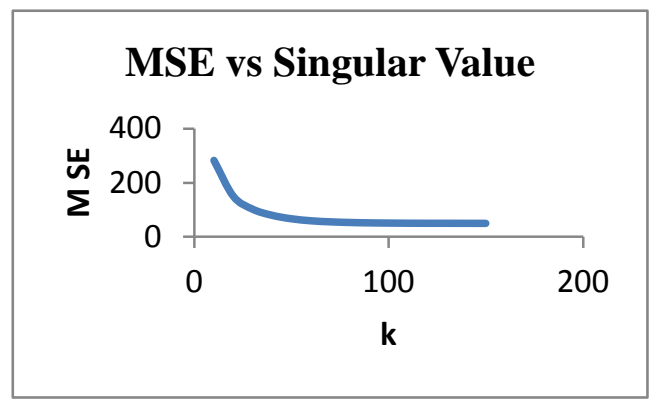

Fig 12- Plot of MSE vs $k$

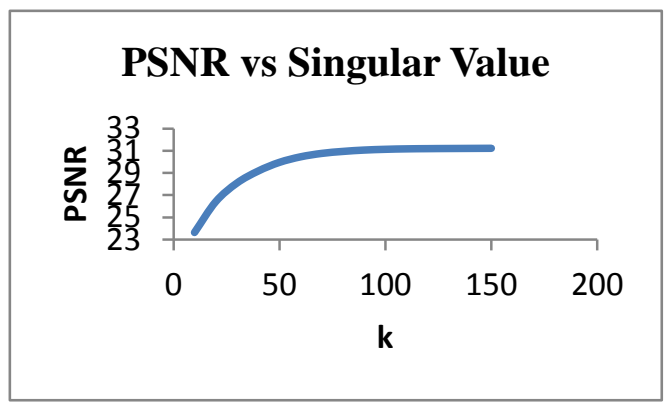

Fig 13- Plot of PSNR vs $k$

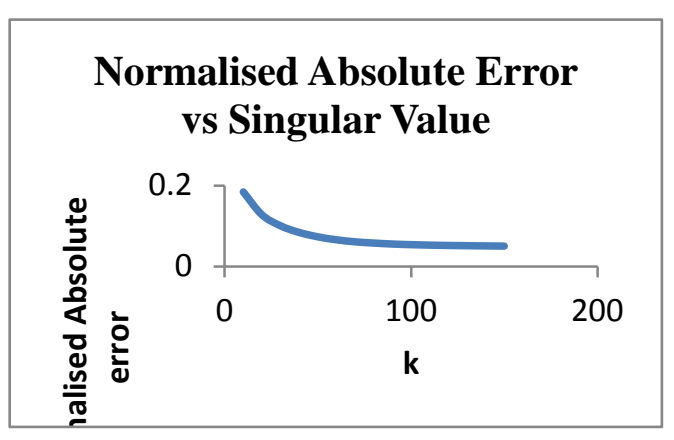

Fig 14- Plot of NAE vs k

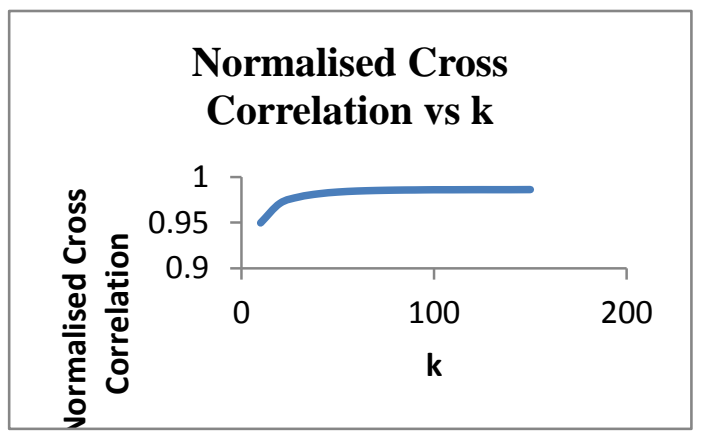

Fig 15- Plot of NC vs k

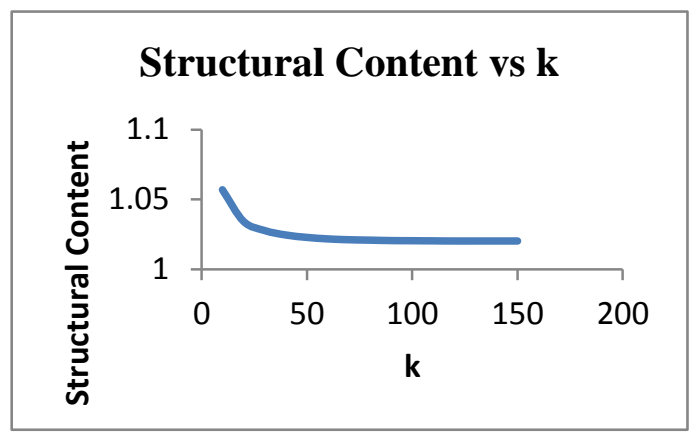

Fig 16- Plot of SC vs k

\section{CONCLUSION}

In this paper, an SVD based fusion approach was implemented, which can be used to fuse two blurred images to increase the clarity of the output image. This is performed by choosing certain components of SVD (U, $\Sigma$ and V) of the input images based on a singular value, and performing fusion of those images. The quality of the images was measured using various image quality measures like PSNR, NC, SC, NAE and MSE. The quality of this fused output keep on increasing as the singular value increases and output images of good quality at higher singular values was obtained. After that SVD based fusion was compared with PCA based fusion. The analysis of image quality measures lead to a conclusion that the proposed approach gives higher quality output in comparison with PCA.

\section{REFERENCES}

[1] Yufeng Zheng, 2011 Image fusion and its applications. INTECH open access publisher

[2] Zhong Zhang and Rick S Blum. 1997 Region based image fusion scheme for concealed weapon detection. Lehigh University, Bethlehem.

[3] Ute G. Gangkofner, Pushkar S. Pradhan and Derrold W. Holcomb 2008. Optimising the highpass filter addition technique for image fusion.

[4] N. Rajic, 2002 Principal component thermography for flaw contrast enhancement and flaw depth characterization in composite structures.

[5] Y. Zheng, et al. 2007 Effective image fusion rules of multiscale imge decomposition

[6] Q.Miao and B. Wang. 2007 A novel image fusion algorithm using FRIT and PCA.

[7] Jing wang, Jimin Liang, Haihong HU, Yan Li Bin Feng. Performance evaluation of infrared and visible image fusion algoriyhm for face recognition. Xidian University, China

[8] Haidawati Nasir, Vladimir Stankovic, Stephen Marshall. 2011 Singular Value Decomposition based fusion for super-resolution image reconstruction. University of Strathclyde

[9] K.P.Soman, R. Loganathan and V.Ajay. Machine learning with SVM and other kernel methods

[10] Manjusha Deshmukh, Udhav Bhosale. Image fusion and image quality assessment of fused images. 
[11] Soumya T Soman, Soumya. V.J, K.P. Soman. 2009 Singular Value decomposition A classroom approach.

[12] Lastman, G.J Sinha. 1986. Use of singular value decomposition in system identification. University of Waterloo.

[13] Zhang, X D Zhang Y. S. 1993. Singular value decomposition-based MA order determination of nongaussian ARMA model.
[14] Andrews, H. Patterson.C. 1976 Singular value decomposition (SVD) image coding.

[15] Shivsubramani Krishnamoorthy, K.P Soman. 2010 Implementation and comparative study of image fusion algorithms. Amrita University 